\title{
Lumbar Cryptorchidism with Splenogonadal Fusion: The Odd Couple
}

\author{
Amol Anil Kulkarni ${ }^{1}$ Ajay S. Kanbur ${ }^{2}$ Nikhil V. Kamat ${ }^{1}$ Lokesh D. Sinha ${ }^{2}$ Prashant Khadse ${ }^{3}$ \\ Riya Prakash Mehta ${ }^{1}$
}

${ }^{1}$ Department of Radiodiagnosis, Jupiter Hospital, Thane, Maharashtra, India

2 Department of Urosurgery, Jupiter Hospital, Thane, Maharashtra, India

${ }^{3}$ Department of Pathology, Jupiter Hospital, Thane, Maharashtra, India

Address for correspondence Amol Anil Kulkarni, DNB, Department of Radiodiagnosis, Jupiter Hospital, Eastern Express Highway, Service Road, Next To Viviana Mall, Thane, Maharashtra 400601, India (e-mail: amolkul93@gmail.com).

J Gastrointestinal Abdominal Radiol ISGAR 2022;5:49-54.

\begin{abstract}
Keywords

- splenogonadal fusion

- bilateral cryptorchidism

- MRI

We present and discuss the clinical, radiological, surgical, and histopathologic findings in a 16-month-old child who presented with bilateral cryptorchidism. He was investigated radiologically with ultrasonography and magnetic resonance imaging, diagnosed with small right inguinal and an enlarged left abdominal testis, and he underwent left orchidectomy and histopathology confirmed the diagnosis of splenogonadal fusion.
\end{abstract}

\section{Introduction}

Splenogonadal fusion (SGF) is a rare congenital anomaly characterized by an abnormal connection between spleen and gonad or another mesonephric derivative during the embryonic period. Less than 200 cases have been reported in the literature. The presentation usually occurs as undescended testis or scrotal mass discovered incidentally during orchidopexy or hernia repair. Preoperative diagnosis is infrequent because of a lack of awareness due to rarity, so if considered in the differential diagnosis may help to prevent unnecessary orchidectomy in select cases. ${ }^{1}$

\section{Case Review}

A 16-month-old child, weighing $7.5 \mathrm{~kg}$, was referred to the urologist for evaluation of bilateral cryptorchidism. He was previously assessed by the same urologist at 4 months of age.

Physical examination showed normal penis and meatus, no hernial swelling, and well-formed scrotal rugae with empty scrotal sacs. Previous ultrasound examination revealed a testis-like structure in the right iliac fossa, and an enlarged left testis in the infrarenal region confirming bilateral undescended testes. His karyotype was 46XY. His testicular tumor markers, namely alpha-fetoprotein (AFP) and human chorionic gonadotropin (hCG), were normal.

Magnetic resonance imaging (MRI) of the abdomen and pelvis with plain computed tomography (CT) cuts was performed.

An oval soft tissue measuring $7 \times 7 \times 5 \mathrm{~mm}$ was seen in the right iliac fossa just above and close to the right deep inguinal ring with partially restricted diffusion suggestive of the undescended right testis. Another ovoid soft tissue with a small accessory appendage measuring $3.3 \times 2.0 \times 1.5 \mathrm{~cm}$ was seen lateral to the lower pole of the left kidney, which appeared hyperintense on T2WI and showed restricted diffusion. No evidence of any other lesions or lymphadenopathy was seen. Findings were suggestive of bilateral undescended testes with possible underlying neoplasm on the left side (-Fig. 1). published online January 25, 2022
DOI https://doi.org/ 10.1055/s-0041-1740477. ISSN 2581-9933. (c) 2022. Indian Society of Gastrointestinal and Abdominal Radiology. All rights reserved.

This is an open access article published by Thieme under the terms of the Creative Commons Attribution-NonDerivative-NonCommercial-License, permitting copying and reproduction so long as the original work is given appropriate credit. Contents may not be used for commercial purposes, or adapted, remixed, transformed or built upon. (https://creativecommons.org/ licenses/by-nc-nd/4.0/)

Thieme Medical and Scientific Publishers Pvt. Ltd., A-12, 2nd Floor, Sector 2, Noida-201301 UP, India 
Being low weight for laparoscopy, he was subjected to open left lumbar orchidectomy through a subcostal incision. At surgery, the suspected left infrarenal tissue was similar in appearance to the spleen which raised the possibility of splenunculus. The operating surgeons were worried about a small ectopic testis in the lower abdomen or pelvis.

A request for retrospective review of MRI images was made during surgery. On review with the background of suspicion for splenunculus, the left infrarenal ovoid tissue showed similar signal intensity to the spleen on all pulse sequences including diffusion-weighted imaging suggestive of splenic tissue (-Fig. 2). A thick connecting cord was identified between the ovoid tissue and spleen, though the cord was difficult to trace among the adjacent bowel loops. The smaller appendage-like structure contiguous to this ovoid tissue was suggestive of an atrophic-ectopic left testis since MRI revealed no other abnormal mass or testicular tissue in other likely ectopic sites. The connecting cord was traced and confirmed on the surgical table (-Fig. 3 ). These combined MRI and surgical findings were indicative of continuous SGF. Left lumbar orchidectomy with splenorrhaphy was performed.

Histopathology showed normal splenic tissue separated from adjacent seminiferous tubules and epididymis, by a capsule and vascular connective tissue confirming SGF (-Fig. 4).

The postoperative period was uneventful. The patient was on regular follow-up and is now planned for right orchidopexy.

\section{Discussion}

SGF is a rare developmental anomaly characterized by an abnormal connection of the splenic and gonadal anlagen. It was first reported by Boestrom in 1883 and less than 200 cases are described so far. The male-to-female ratio is approximately 16:1 and occurs mostly on the left side (98\%). Patients may present with a scrotal mass or are detected incidentally during surgery for cryptorchidism or inguinal hernia repair. Unusual presentations include bowel obstruction by the intraperitoneal cord, traumatic rupture of ectopic splenic tissue, association with intra-abdominal seminoma, and nonseminomatous tumor. Diagnosis can occur at any age; however, in most reported cases the patients are younger than 30 years and more than half of them were seen before the age of 10 years. ${ }^{1,2}$

The spleen develops from the dorsal mesogastrium around the 5 th week of gestation as a proliferation of surface

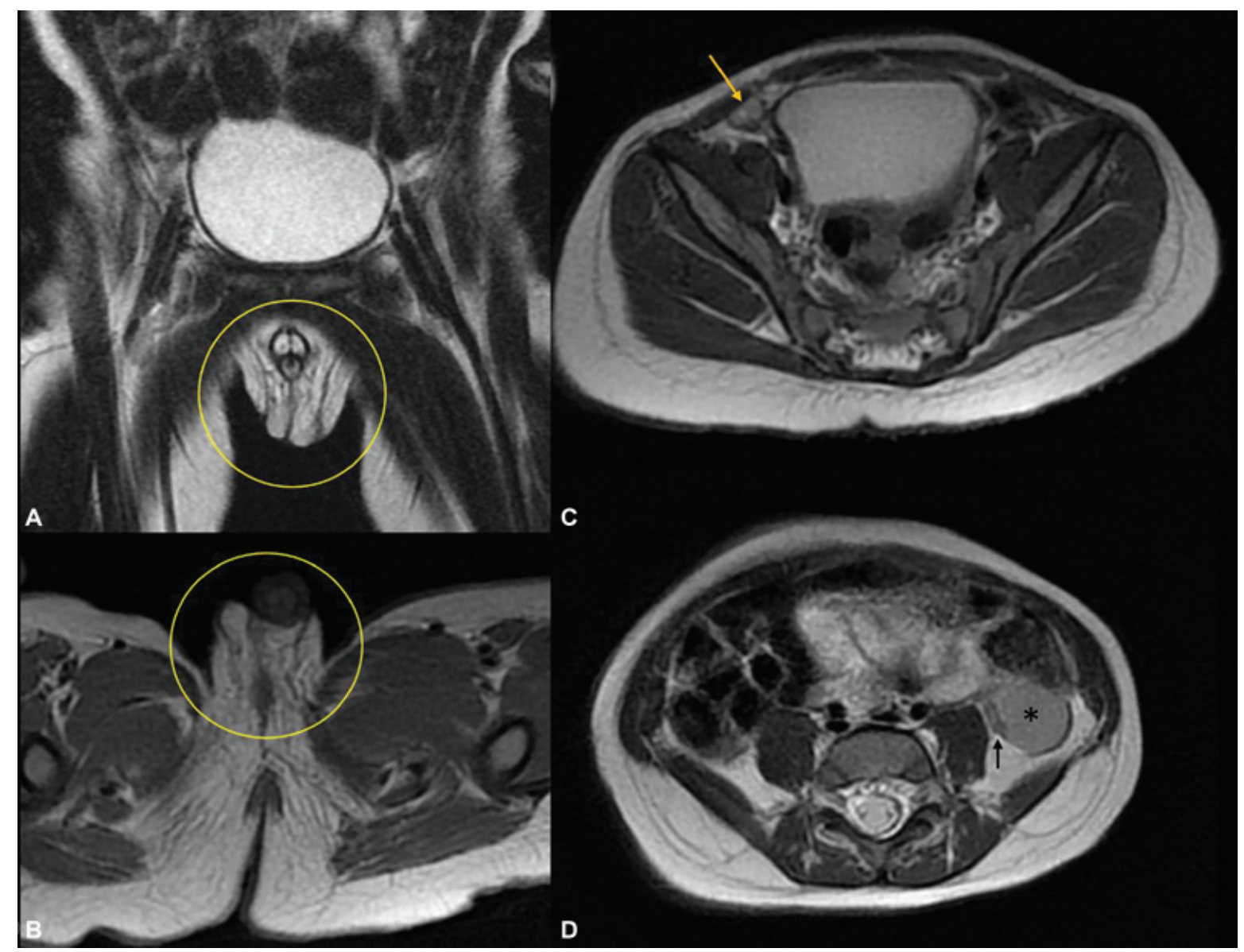

Fig. 1 (A) Coronal T2WI and (B) axial T1WI showing bilateral empty scrotal sacs. (C) Axial T2WI showing right testis at deep inguinal ring. (D) Axial T2WI showing ovoid soft tissue in left infrarenal region-asterisk-and accessory appendage-like structure-black arrow. 


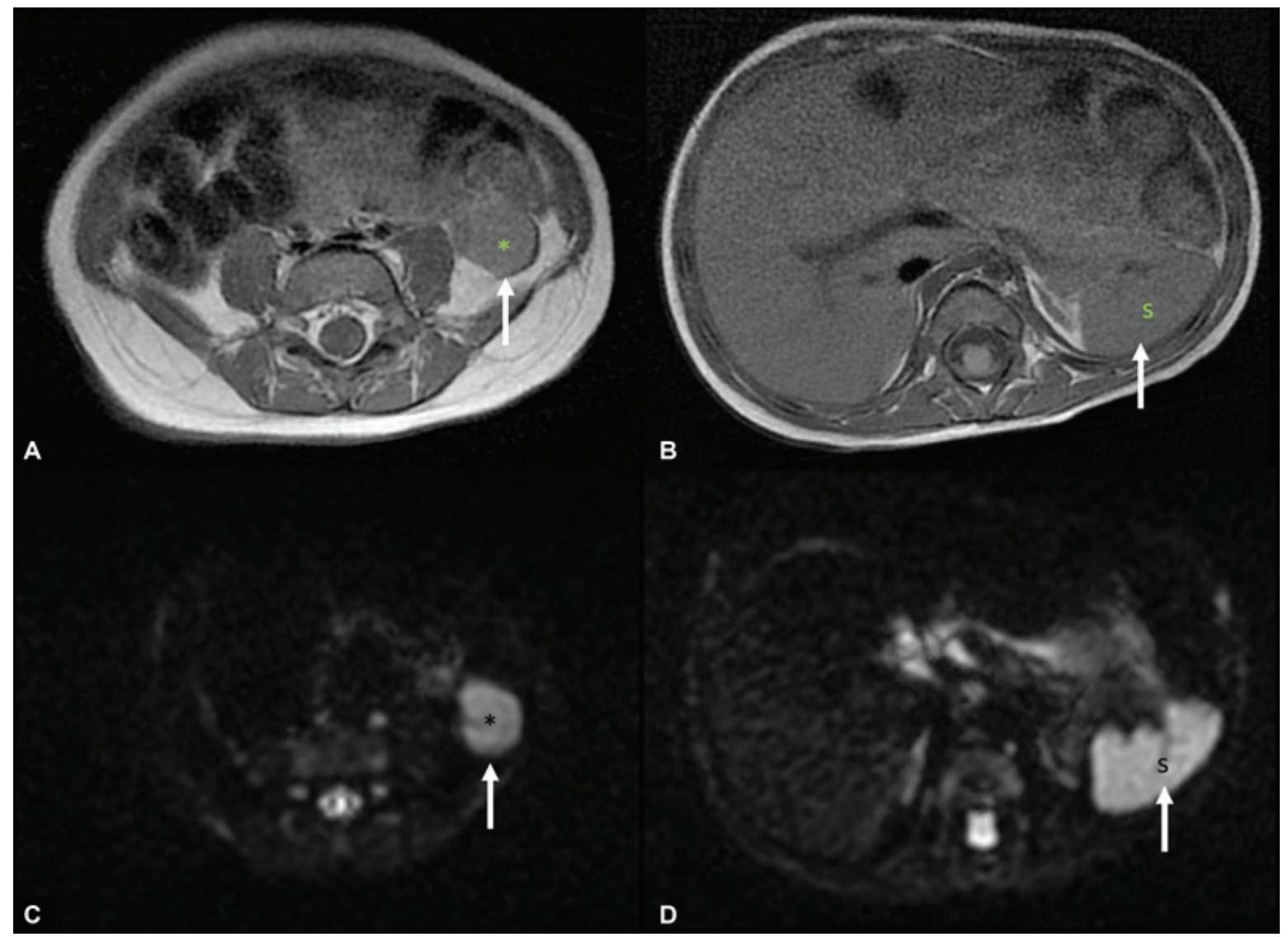

Fig. 2 (A, B) Axial T1WI and (C, D) diffusion-weighted imaging (DWI) showing S-spleen-and asterisk-ovoid soft tissue showing similar signal intensities possibly suggesting same origin.

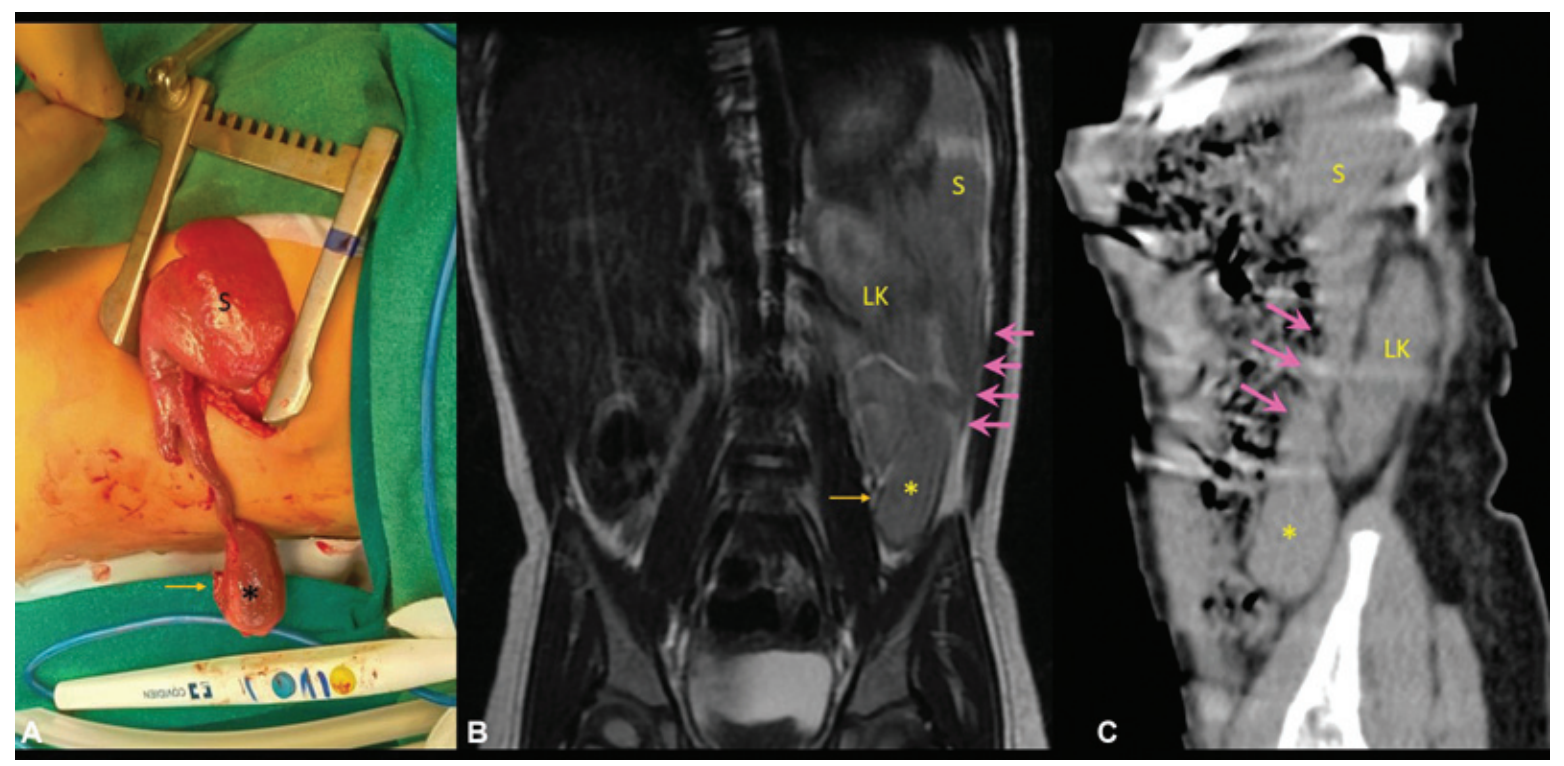

Fig. 3 (A) Intraoperative image showing direct connection between spleen (S) and ovoid tissue $\left({ }^{*}\right.$ ) with a small appendage-like testis (yellow arrow). (B) Coronal T2WI and (C) sagittal reformatted computed tomography (CT) images showing direct connection between spleen (S) and ovoid tissue $(*)$ by a connecting cord (pink arrows) and small testis (yellow arrow). 


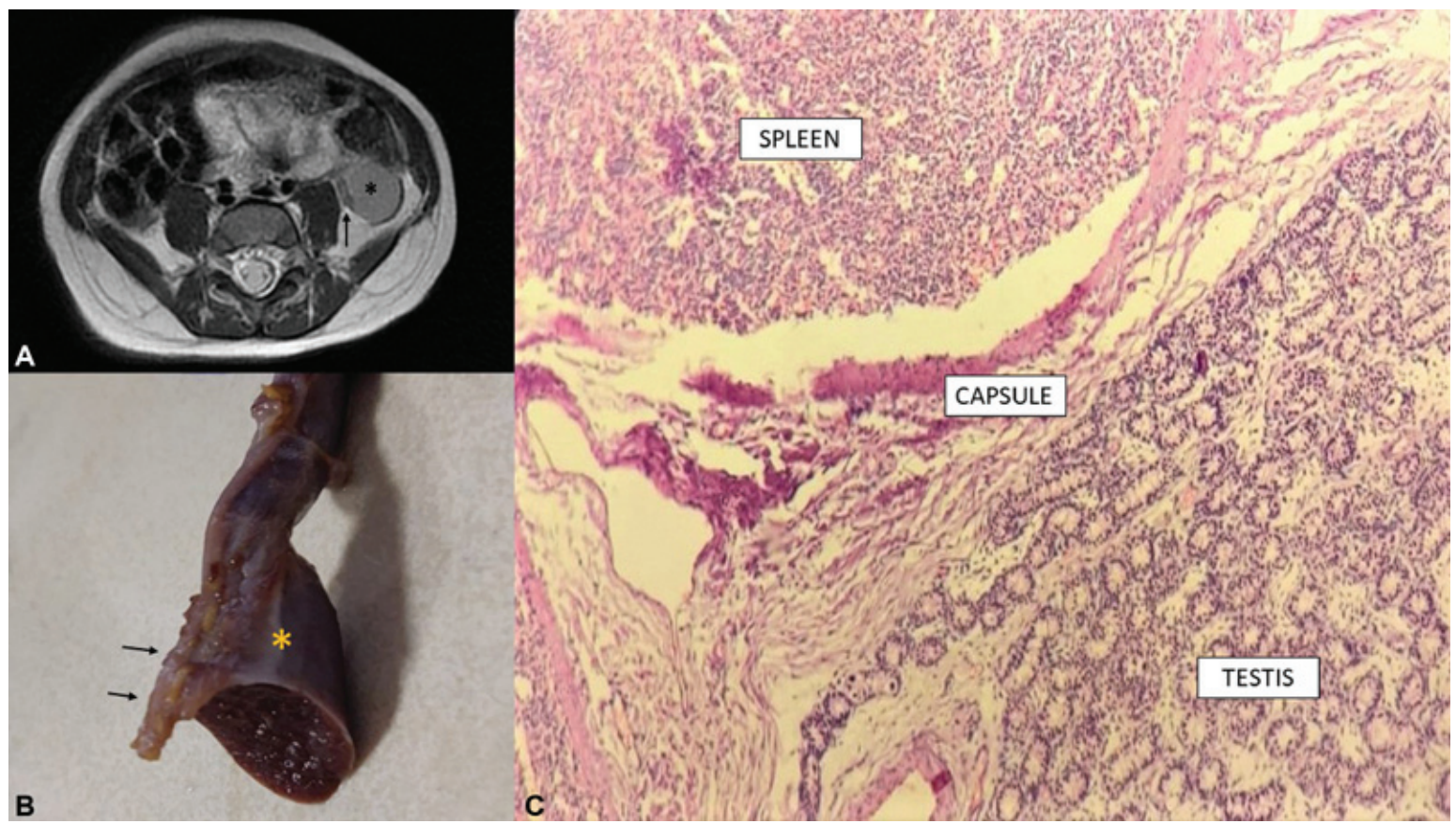

Fig. 4 (A) Axial T2WI showing testis: black arrow, and ovoid soft tissue in left infrarenal region: asterisk. (B) Gross specimen of the excised tissue showing linear testicular tissue: black arrows, and ovoid splenic tissue: yellow asterisk. (C) Histopathology slide showing adjacent splenic and testicular tissue separated by a capsule.

peritoneal cells on the left side. As the spleen develops it rotates into proximity with the gonadal ridge and fusion probably occurs during the 5 th to 8 th week of gestation. The gonadal descent occurs during 8 to 10 weeks, and the splenic tissue could be found anywhere along the pathway of descent sometimes seen as a band of tissue/connecting cord (fibrous or splenic). The initiating events of the fusion are currently unknown, it has been hypothesized that a mild inflammation of the peritoneal surfaces leads to adhesions or lack of apoptosis at the interface between splenic primordium and continuous genital ridge may occur (-Fig. 5). ${ }^{1-4}$

SGF has been classified into two types by Putschar and Manion. The continuous type shows the uninterrupted connection of the spleen and the gonad by a cord-like tissue. The discontinuous type has no connection between the spleen and gonad. The development of limb buds and mandible occurs during the same time, accounting for the close association of SGF with malformations of limbs and mandible (SGF limb defect syndrome) (-Table 1). ${ }^{1-5}$

Ultrasound is the usual first-line investigation for the evaluation of cryptorchidism. It is widely available and does not involve radiation nor requires sedation. In cases of discontinuous SGF, we may see a hypoechoic extratesticular scrotal mass with an echogenic capsule. Color Doppler imaging may demonstrate internal vascularity with a branching pattern different from the "criss-cross" pattern of a malignancy. With cases of continuous SGF, the ultrasound features are similar with an additional demonstration of connecting the cord between the spleen and testis. ${ }^{2,4,5}$

CT was initially believed to be helpful in the diagnosis of SGF. Multiphasic contrast-enhanced CT including angiographic and venous phases demonstrates vascularity and may aid in diagnosis by identifying gonadal vein. In an appropriate clinical scenario, a homogenous, noncalcified enhancing soft tissue density mass with attenuation similar to the spleen may suggest SGF. But CT involves ionizing radiation thus should be used judiciously in younger radiosensitive patients. ${ }^{4,5}$

MRI may be considered as an investigation of choice for the evaluation of cryptorchidism as well as SGF. It does not involve radiation and can demonstrate similarities of signal characteristics between the spleen and testis as well as demonstrate the connecting cord. ${ }^{2}$

99m Tc-sulfur colloid liver spleen scintigraphy demonstrates uptake in splenic tissue. ${ }^{2,5}$

SGF is rare, hence a relatively unknown entity, and the radiological diagnosis is ambiguous. Diagnosing SGF is a formidable task because it lacks characteristic clinical features (-Table 1). Presentation as a scrotal mass mimics neoplasm and the SGF diagnosis is often overlooked and may result in male patients undergoing unnecessary orchidectomy. ${ }^{1-5}$ Awareness of the entity and appearance of the mass lesion showing similar characteristics of the spleen can be a pointer to the condition. SGF with cryptorchidism may be associated with malignancy (cryptorchidism is the presumed causative factor for associated malignancy in cases of SGF). Tumor biomarkers (AFP, $\beta$-hCG, carcinoembryonic antigen, and lactate dehydrogenase) play an important role to rule out testicular tumors. ${ }^{1,5,6}$

Treatment of SGF is controversial. Surgery may not be required for scrotal SGF or if performed testis could be preserved. If the SGF shows no signs of inflammation, the splenic tissue can be easily dissected from the gonadal structures, and the testis can be preserved. Laparoscopic 

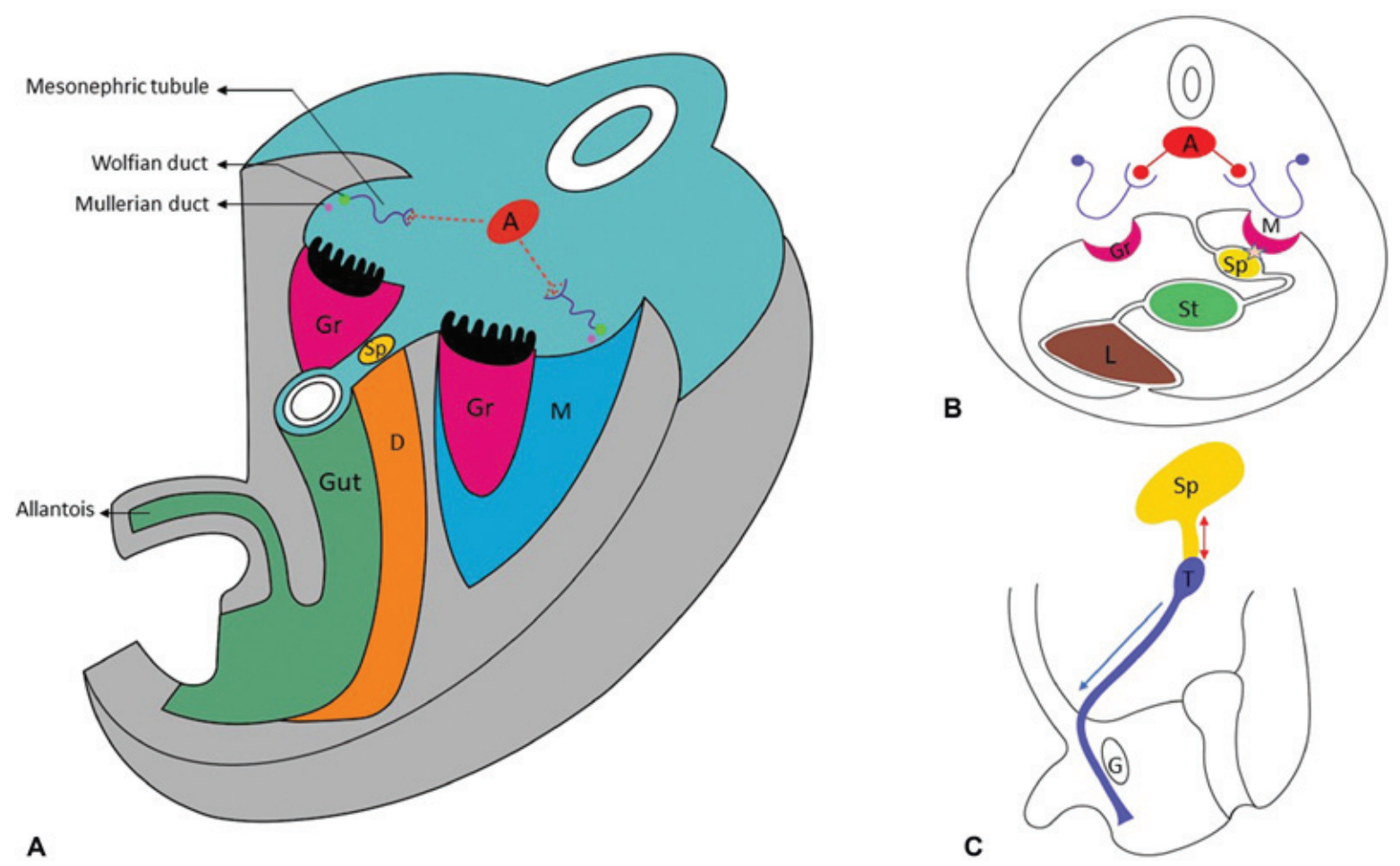

Fig. 5 Embryology of splenogonadal fusion. (A) The spleen (Sp) develops within the dorsal mesogastrium (D) as condensation of splenic anlage mesoderm. (B) Fusion (orange star) of the developing spleen (Sp) to the gonadal ridge (Gr) during rotation. (C) Testicular descent (blue arrow) with descent of a portion of the spleen (red double-headed arrow) along with it. A, aorta; G, gubernaculum; Gr, gonadal ridge; L, liver; M, mesonephros; St, stomach; $t$, testis. Image courtesy: Varma at al. ${ }^{4}$

Table 1 Types and features of splenogonadal fusion ${ }^{1-3}$

\begin{tabular}{|c|c|c|}
\hline & SGF continuous type & Discontinuous type \\
\hline Definition & Connecting cord between spleen and gonad & $\begin{array}{l}\text { No connection between spleen and gonad, } \\
\text { or ectopic splenic tissue }\end{array}$ \\
\hline Incidence & $\begin{array}{l}55 \% \\
\text { Most cases in females are of continuous type }\end{array}$ & $45 \%$ \\
\hline Associated Malformations & $\begin{array}{l}5 \text { times more common than discontinuous type } \\
\text {-cryptorchidism (most common) } \\
\text {-others; limb bud deformities, micrognathia, } \\
\text { and cardiac anomalies }\end{array}$ & Uncommon \\
\hline \multirow[t]{2}{*}{ Imaging } & Demonstration of the connecting cord & $\begin{array}{l}\text { No connection, ectopic scrotal tissue } \\
\text { mimics neoplasm }\end{array}$ \\
\hline & \multicolumn{2}{|c|}{$\begin{array}{l}\text { USG: Hypoechoic mass compared with testis separated by an echogenic capsule and } \\
\text { shows branching internal vascularity on Doppler } \\
\text { CT: Homogeneous, noncalcified enhancing soft tissue with attenuation similar to the } \\
\text { spleen } \\
\text { MRI: Similar signal characteristics of spleen and suspected tissue }\end{array}$} \\
\hline Tc-99m & \multicolumn{2}{|l|}{ Similar uptake in spleen and suspected tissue } \\
\hline
\end{tabular}

Abbreviations: CT, computed tomography; MRI, magnetic resonance imaging; SGF, splenogonadal fusion; USG, ultrasonography.

exploration and perioperative frozen sections may be performed to rule out malignancy, thus preventing unnecessary orchidectomy.

SGF associated with cryptorchidism increases the risk of malignancy and hence orchidectomy is a better option. ${ }^{6}$

\section{Conclusion}

SGF, located in the scrotum or at other ectopic locations, is a very rare entity and often misdiagnosed as malignancy. A high index of suspicion and a multidisciplinary approach to 
54 Lumbar Cryptorchidism with Splenogonadal Fusion Kulkarni et al.

the investigation is advocated to accurately diagnose this anomaly for appropriate treatment.

\section{Conflict of Interest}

None declared.

\section{References}

1 Huang G, Huang Y, Zeng L, Yuan M, Wu Y, Huang L. Continuoustype splenogonadal fusion: a case report. Exp Ther Med 2017;13 (05):2019-2021

2 Foellings K, Baker S, Walker D. Sonographic and magnetic resonance imaging characteristics associated with Splenogonadal fusion. J Diagn Med Sonogr 2014;30:256-260
3 Duhli N, Venkatramani V, Panda A, Manojkumar R. Splenogonadal fusion: pathological features of a rare scrotal mass. Indian J Pathol Microbiol 2013;56(04):474-476

4 Varma DR, Sirineni GR, Rao MV, Pottala KM, Mallipudi BV. Sonographic and CT features of splenogonadal fusion. Pediatr Radiol 2007;37(09):916-919

5 Jakkani R, Alhajri FA, Alteriki A, Almuteri MF, Athyal RP, Hashem KZ. Discontinuous splenogonadal fusion diagnosed on computed tomography. Indian J Radiol Imaging 2016;26 (04):506-509

6 Patil SV, Patil PS, Sharma VD, Jadhav SS. Splenogonadal fusion a rare anomaly. J Indian Assoc Pediatr Surg 2020;25(02): $115-117$ 